\title{
Psychological CAREER RESOURCES OF WORKING ADULTS: a South African survey
}

\author{
MELINDE COETZEE \\ Department of Industrial \& Organisational Psychology \\ University of South Africa \\ South Africa \\ Correspondence to: Melinde Coetzee \\ e-mail:coetzm1@unisa.ac.za
}

\begin{abstract}
The main objective of this study was to explore broad trends regarding how individuals from various age, educational, marital, race and gender groups in the South African organisational context differ in terms of their psychological career resources, as measured by the Psychological Career Resources Inventory. A sample of 2997 working adults registered as students at a South African higher distance education institution participated in this study. The results indicate significant differences between the various biographical variables and the participants' psychological career resources. In the context of employment equity, and with more women entering the workplace, this study is expected to contribute important knowledge that will inform career development practices concerned with enhancing employees' career meta-competencies as an important element of their general employability.
\end{abstract}

Keywords: career drivers, career enablers, career harmonisers, career meta-competencies, career preferences

In recent years, employees' employability has become a major concern for organisations. Employability is a critical requirement for sustaining a competitive advantage at company level and career success at individual level (Van Dam, 2004; Van der Heijde \& Van der Heijden, 2006). In the context of the 21st century people are regarded as competency traders and their employability depends on their knowledge, transferable skills, experience, unique attributes and accomplishments. Employability therefore refers not only to people's ability to gain access to the workplace, to adjust to the workplace, and to be productive in the workplace, but also to their continuous ability to fulfil, acquire, or create work through the optimal use of both occupation-related and career meta-competencies (Coetzee \& Roythorne-Jacobs, 2007; Hall \& Chandler, 2005; Herr, Cramer \& Niles, 2004; Van der Heijde \& Van der Heijden, 2006). Career meta-competencies refer to skills and abilities such as behavioural adaptability, identity awareness, sense of purpose, self-esteem and emotional intelligence, which enable people to be self-directed learners and proactive agents in the management of their careers (Briscoe \& Hall, 1999; Coetzee \& Roythorne-Jacobs, 2007; Hall \& Chandler, 2005; Herr et al., 2004). People's career meta-competencies facilitate the acquisition of other, more specific competencies or skills which promote both their general employability and occupational expertise (Briscoe \& Hall, 1999; Hall \& Chandler, 2005).

Moreover, in an increasingly turbulent environmental context people are less dependent on organisational career arrangements because they experience more frequent career transitions, have greater agency in career decisions and must be able, adaptable learners (Arthur, Khapova \& Wilderom, 2005; Baruch, 2004). Consequently, subjective career measures that emphasise psychological attributes and abilities have taken on greater salience in today's work environment (Brown, GeorgeCurran \& Smith, 2003; Ebberwein, Krieshok, Ulven \& Prosser, 2004; Kidd, 2004; Van Dam, 2004). More and more researchers in the field of career development therefore suggest that greater awareness of the effect of individuals' occupational expertise and their career meta-competencies (as indicated by an individual's range of psychological career resources) on their general employability may become a more important function than career development planning in the present era of fast technological changes, globalisation, demographic workforce changes and changing organisational structures (Coetzee \& Roythorne-Jacobs, 2007; Gowan, Craft \& Zimmerman, 2000; Hall \& Chandler, 2005; Herr et al., 2004; Kim, 2005; Savickas, 2000; Van Dam, 2004; Van der Heijde \& Van der Heijden, 2006).

\section{Psychological career resources}

In the context of this study, the term psychological career resources is defined as the set of career-related orientations, values, attitudes, abilities and attributes that lead to selfempowering career behaviour and promote general employability (Coetzee \& Roythorne-Jacobs, 2007). Individuals repertoires of psychological career resources consist of those career preferences, career values, attributes, skills and attitudes that can be linked to their experiences of intrinsic or subjective career success (Gunz \& Heslin, 2005). Psychological career resources are therefore also regarded as individuals' inherent resources or meta-competencies which enable them to adapt to changing career circumstances and to shape and select environments in order to attain success within a particular socio-cultural context (Ebberwein et al., 2004; Gowan et al., 2000; Sternberg, 1999).

Coetzee (2006) provides a useful theoretical framework to help individuals understand the importance of developing their internal career resources and tapping into these psychological resources to enhance their general employability attributes and skills (which usually result in subsequent experiences of intrinsic career satisfaction). Figure 1 shows how the various psychological career resources fit together.

As depicted in Figure 1, people's career preferences and career values (people's unique views about the paths their careers should follow) guide their career decisions. The drivers (people's sense of career purpose, career directedness and career venturing attitude) are the attitudes that energise people and motivate them towards experimenting with career and employment possibilities that are based on their viewpoints of the possible selves they could become or the possible working roles they could experience. The enablers (people's transferable skills such as their practical or creative skills and self/other skills) are those abilities that help them to succeed in their careers. The harmonisers (people's self-esteem, behavioural adaptability, emotional literacy and social connectivity) are the psychological 


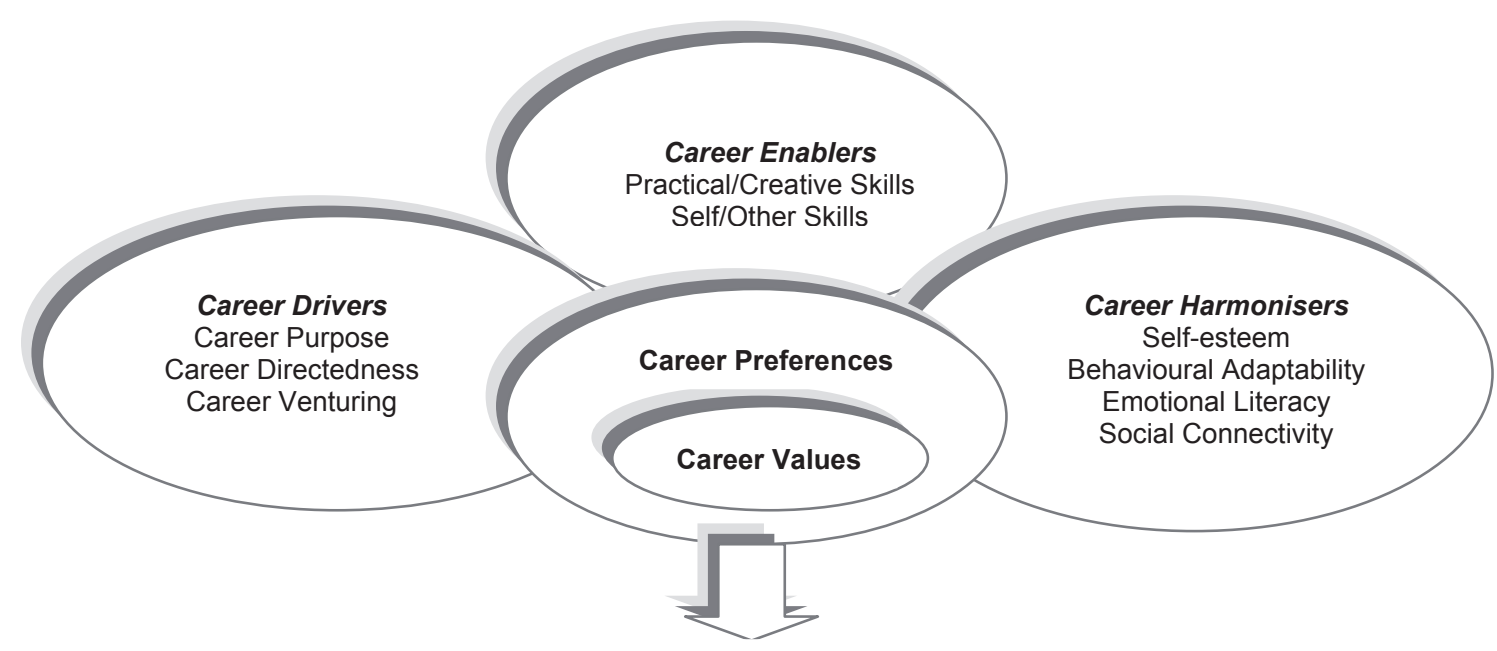

Continuously reinventing one's career identity, the form and shape of one's career

FIGURE 1

A theoretical framework of the construct psychological career resources (based on Coetzee, 2006)

attributes which not only act as promoters of flexibility and resiliency, but also as controls by keeping the career drivers in balance so that people do not go overboard (or burn themselves out) in the process of pursuing and reinventing their careers (Coetzee \& Roythorne-Jacobs, 2007).

\section{Career preferences and career values}

Individuals' career preferences and career values are regarded as the enduring cognitive or conceptual structures underlying their thoughts about their careers and which define the meaning of a career to them (Driver, 1982; Kim, 2005). Career preferences and career values become the guiding foundation for individuals' long-term career choices. Whereas individuals' career preferences guide their career moves, career values represent the motivation for a particular career preference (Brousseau, 1990). Based on the career orientation models of Driver and Brousseau (1988) and Derr (1986), Coetzee (2007) identifies four career preferences in terms of the psychological career resources framework, namely: stability/expertise, managerial, creativity/variety and autonomy/independence.

The stability/expertise career preference refers to individuals' orientation towards occupations or jobs that offer stability, predictability and the opportunity to develop their expertise in a particular field (Brousseau, 1990; Derr, 1986). While the managerial career preference refers to individuals who view upward mobility to positions of successively greater responsibility, influence and authority as career success, individuals with autonomy/independence as a career preference regard independence and freedom from external interruptions as the ideal work situation (Derr, 1986). Both the managerial and autonomy/independence career preferences have been found to be significantly associated with the need for authority and influence as a career motive (Coetzee, 2007). The creativity/variety career preference describes individuals' need for a career that allows them to work on a variety of different tasks that require them to use and develop a wide range of skills, abilities and knowledge in innovative and creative ways (Brousseau, 1990). Research findings by Coetzee (2007) indicate that the stability/expertise and creativity/variety career preferences are significantly associated with individuals' needs for further growth and development as a career value.

\section{Career drivers}

In terms of career drivers, the term career purpose refers to individuals' sense of having a career calling. People's career calling often involves a self-reflective quest for a personal and professional goal. This sense of higher purpose is based on people's confidence and inner conviction that they are able to achieve their career goals which include being of service to others and giving their best in what they do (Hall \& Briscoe, 2004; Hall \& Chandler, 2005; Hansen, 1997). Wrzesniewski, McCauley, Rozin and Schwartz (1997) state that people who feel strongly called to their careers and jobs report much higher levels of life and job satisfaction and lower levels of absenteeism than people who have a mere job and/or career orientation. People with a calling orientation (or strong sense of purpose) tend to work for the fulfilment their jobs and careers offer and, in addition, believe that their work impacts society in some way (Bellah, Sullivan, Tipton, Madsen, \& Swindler, 1996). Career directedness is described as individuals' sense of clarity about future career directions and goals as well as the clarity in terms of where and how to find support for achieving their career goals or finding new job opportunities. The term career venturing relates to individuals' willingness to take risks in finding and experimenting with new career opportunities (Coetzee, 2007).

According to Weiss, Skelley, Freund, Haughey and Hall (2004), people's sense of purpose and career directedness is expressed through the use of their strengths and aptitudes. Individuals are drawn to enact their careers using abilities which represent their genius. Therefore individuals will pursue goals and task efforts that use their greatest strengths. In addition, when facing unfamiliar or difficult task situations, individuals with a sense of purpose will more likely be able to manage temporary setbacks or failures and be willing to venture out in search of new opportunities because they will tend to believe that ultimately they will succeed (Hall \& Chandler, 2005; Weiss et al., 2004). Research in the field shows that whereas task success can lead to experiences of psychological failure, strategies of proactive life and career management, such as a strong sense of purpose, commitment to personal goals and focusing psychological resources and effort predicted how emotionally balanced and satisfied people would feel three years later (Wiese, Freund \& Baltes, 2002). 
Career enablers

Based on Sternberg's (1985) and Gardner's (1983) notion of multiple intelligences, Coetzee (2007) identified two constructs related to the concept of career enablers, namely practical/ creative skills and self/other skills. Sternberg (1985) describes practical intelligence as the skill or ability to apply one's intelligence to real-world contexts. Creative intelligence is described as individuals' ability to apply cognitive processes in challenging existing assumptions and concentrating on new ways of doing things. In the context of careers, both practical intelligence and creative intelligence are required to implement career options and to make them work in innovative and creative ways (Bay \& Lim, 2006; Coetzee, 2007). The term self/other skills relates to Gardner's (1983) notion that personal intelligence consists of interpersonal intelligence (or the ability to understand, honour, empathise and interact effectively with others) and intrapersonal intelligence (or the ability to understand one's feelings and motivations and exerting selfdiscipline in one's interactions) (Bay \& Lim, 2006; Coetzee, 2007).

Gardner (1983) states that the development of self-other intelligence is important as individuals function within a social context. It is within the social context that individuals develop their sense of self which creates the balance that they strike between their feelings and inner voices, and the expectations of others. Careers are also increasingly regarded as social processes. This leads researchers to attend more to the role of emotions and feelings in the career decision-making process (Kidd, 2004). Studies by Phillips, Christopher-Sisk and Gravino (2001) and Higgins (2001) showed that career decision making takes place in a relational context. Research by Emmerling and Cherniss (2003) indicated that the emotions experienced during the career decision-making process and in relation to social relations at work have implications for the perception of risk related to specific career options, the amount and kind of selfexploration individuals will engage in, and how information related to career choice will be processed.

\section{Career harmonisers}

Individuals' emotional literacy and social connectivity are regarded as the core aspects that underlie the construct career harmonisers (Coetzee, 2007). Emotional literacy refers to the degree to which individuals are able to accept and express a range of affect, based on the premise that a range of emotional responses facilitates career adaptive behaviours in the career decision-making process (Emmerling \& Cherniss, 2003). Emotions are adaptive when they prioritise thinking in a way that ensures that the individual attends to the most important or distressing aspects of a decision. On the other hand, the anxiety that is often associated with career indecision can be viewed as maladaptive (Crites, 1974). Social connectivity describes individuals' ability to connect with others, and establish and maintain mutually satisfying and supporting relationships (BarOn, 1997). Ensel and Lin (1991) and Hammer (1988) report findings which suggest that being embedded in social networks safeguard or buffer people against the negative impact of psycho-social stressors on their physical health.

Coetzee (2007) views individuals' behavioural adaptability and self-esteem as two additional attributes that form an integral part of the construct career harmonisers. Behavioural adaptability involves individuals' ability to identify those qualities that are critical for future performance and being able to make the necessary personal changes to meet their career-related needs (Hall, 2002). Thus, individuals with high behavioural adaptability would have the capacity to engage proactively in the process of goal setting, initiating effort and achieving psychological success (Hall \& Chandler, 2005). The term self-esteem refers to the self-evaluations people make and maintain. It includes attitudes of approval or disapproval and the degree to which people feel worthy, capable, significant and effective in comparison to other members of their social group (Battle, 1992; Hewitt, 2002). Positive self-esteem is indicative of a positive and integral personal and social identity, that is, a sense that one is located securely in the social world, competent to meet its challenges, ready to participate in life with others, and able to balance social demands and personal desires (Garrety, Badham, Morrigan, Rifkin, \& Zanko, 2003).

Individuals' self-esteem, emotional literacy and social connectivity promote their behavioural adaptability and help them to gain self-confidence in their ability to successfully perform a particular task (BarOn, 1997; Coetzee \& RoythorneJacobs, 2007; Hollenbeck \& Hall, 2004). According to Coetzee (2007), the various components of an individual's psychological resource repertoire need to be in balance to enable the development of the whole person. If any one facet is out of balance, none of the other components can function at its maximum potential to facilitate self-empowering career behaviour. Balanced, optimal functioning of the psychological resources is an indication of self-directed career behaviour that is internally guided and driven by the individual's career preferences, career values, sense of purpose, career directedness and self-awareness

\section{Research objective}

Research (Brown et al., 2003; Coertse \& Scheepers, 2004; Gowan et al., 2000; Ebberwein et al., 2004; Kim, 2005; Lounsbury, Loveland, Sundstrom, Gibson, Drost, \& Hamrick, 2003; Van Dam, 2004) has revealed relationships between psychological career resources variables such as career orientations, personality attributes, emotional intelligence, career resiliency and career maturity, and several career-related variables such as career adaptability, career satisfaction, career decision making, career planning, career initiative, employability and entrepreneurial activity. Findings from these researchers also indicate differences between various cultural, race and gender groups regarding these career variables and experiences of subjective career satisfaction.

The main objective of this study was therefore to explore broad trends regarding how individuals from various age, educational, marital, race and gender groups in the South African organisational context differ in terms of their psychological career resources. In the context of employment equity, and with more women entering the workplace, this study is expected to contribute important knowledge that will inform career development practices concerned with enhancing employees' career meta-competencies as an important element of their general employability.

\section{RESEARCH DESIGN}

\section{Research approach}

For this exploratory pilot study, a survey design was used to achieve the research objective (Shaughnessy \& Zechmeister, 2003). By applying the Psychological Career Resources Inventory (PCRI) and a biographical questionnaire, each participant was measured at a particular point in time in terms of the psychological career resources construct and the participant's biographical variables. The relationship between the measurements was then determined. The advantages of the survey research approach include savings on time and money, an absence of interviewer bias, accurate results, more privacy for participants, and the fact that samples need not be very large in relation to the population (Salkind, 1997). The major disadvantage of this design is that findings can only be generalised to the sampled population at the time of the survey (Dooley, 1995). 
TABLE 1

Reliability statistics: PCRI scales ( $\mathrm{N}=2$ 997)

\begin{tabular}{lllll}
\hline $\begin{array}{l}\text { PCRI } \\
\text { SCALE }\end{array}$ & $\begin{array}{l}\text { NUMBER } \\
\text { OF ITEMS }\end{array}$ & $\begin{array}{l}\text { CRONBACH'S } \\
\text { ALPHA }\end{array}$ & MEAN & SD \\
\hline Career Preferences & & & & \\
\hline Stability/Expertise & 6 & 0.73 & 21.13 & 2.911 \\
Managerial & 4 & 0.75 & 11.37 & 3.071 \\
Variety/Creativity & 3 & 0.70 & 9.52 & 2.141 \\
Freedom/Autonomy & 4 & 0.62 & 11.25 & 2.806 \\
Scale overall & $\mathbf{1 7}$ & $\mathbf{0 . 8 4}$ & $\mathbf{5 3 . 2 8}$ & $\mathbf{8 . 2 2 1}$ \\
Career Values & & & & \\
\hline Growth/Development & 5 & 0.74 & 17.91 & 2.324 \\
Authority/Influence & 3 & 0.61 & 8.52 & 2.139 \\
Scale overall & $\mathbf{8}$ & $\mathbf{0 . 7 4}$ & $\mathbf{2 6 . 4 3}$ & $\mathbf{3 . 7 5 7}$ \\
Career Enablers & & & & \\
\hline Practical/creative skills & 4 & 0.68 & 9.65 & 2.764 \\
Self/other skills & 4 & 0.63 & 13.61 & 2.100 \\
Scale overall & $\mathbf{8}$ & $\mathbf{0 . 7 1}$ & $\mathbf{2 3 . 2 5}$ & $\mathbf{4 . 0 1 5}$ \\
Career Drivers & & & & \\
\hline Career Purpose & 5 & 0.66 & 18.11 & 2.066 \\
Career Directedness & 3 & 0.63 & 9.01 & 2.028 \\
Career Venturing & 2 & 0.70 & 5.84 & 1.707 \\
Scale overall & $\mathbf{1 0}$ & $\mathbf{0 . 7 8}$ & $\mathbf{3 2 . 9 6}$ & $\mathbf{4 . 5 9 7}$ \\
Career Harmonisers & & & & \\
\hline Self-esteem & 7 & 0.77 & 23.08 & 3.605 \\
Behavioural adaptability & 6 & 0.73 & 19.33 & 3.233 \\
Emotional literacy & 5 & 0.70 & 15.28 & 3.008 \\
Social connectivity & 3 & 0.67 & 10.00 & 1.655 \\
Scale overall & $\mathbf{2 1}$ & $\mathbf{0 . 8 8}$ & $\mathbf{6 7 . 6 9}$ & $\mathbf{9 . 2 0 8}$ \\
\hline & & & & \\
\hline & & & & \\
\hline
\end{tabular}

\section{Participants}

The participants were a random sample of employed students who were registered across various fields of studies at a higher distance education institution for a particular year. The total sample of 2997 students constituted 58\% females and $42 \%$ males. Blacks represented $67 \%$ and whites $33 \%$ of the sample. The sample was represented by predominantly Nguni- and Sotho-speaking Africans (41\%) followed by white Englishand Afrikaans-speaking participants (31\%). English-speaking Indians, English- and Afrikaans-speaking coloureds and other minority cultural groups speaking languages such as Portuguese, Germa, Polish, French and Chinese were underrepresented. The sample was predominantly represented by mostly single (52\%) and married $(42 \%)$ participants in the early adulthood life stage (25-40 years) $(82 \%)$. The mean age of participants was 32, which implies well-established internal career preferences and values (Schein, 1996).

The sample had a relatively high educational level, with $84 \%$ having attained a Grade 12 qualification, diploma and undergraduate higher education qualification. The sample predominantly represented participants in full-time employment $(80 \%)$, who occupied relatively high-level positions at senior and middle management level $(18 \%)$ and middle- and first-level supervisory level (54\%) in the service industry $(81 \%)$ with occupational expertise predominantly in the financial $(21 \%)$, education $(11 \%)$, human resource management $(9 \%)$, protective services $(9 \%)$, and health care $(8 \%)$ fields.

\section{Measuring instruments}

A biographical questionnaire and the Psychological Career Resources Inventory (PCRI) developed by Coetzee (2007) were used to measure the variables of concern to this study.

\section{Biographical Questionnaire}

The influence of demographic variables on careers has been examined in a variety of studies (Allen \& Katz, 1992; Erdoğmus, 2003; Igbaria, Greenhaus, \& Parasuraman, 1991; Marshall \&
Bonner, 2003; Schneer \& Reitman, 1994). In the light of the literature, the questionnaire for this study included a series of self-reported questions in order to obtain biographical data such as age, gender, race, home language, marital status, educational level, field of occupational expertise, occupational title and position and economic sector. These data were used to examine relationships between biographical information and the participants' psychological career resources.

\section{Psychological Career Resources Inventory (PCRI)}

The PCRI (Coetzee 2007) is a self-rated multi-factorial measure which contains 64 items and five subscales (career preferences, career values, career enablers, career drivers, career harmonisers). To avoid neutral answers, a 4-point Likert-type scale was used for subject responses to each of the 64 items. Based on Coetzee's (2007) psychological career resources model, the items related to perceived agreement of statements. The PCRI measures 15 constructs in total. The various constructs measured by each of the subscales are shown in Table 1. Results of Exploratory Factor Analyses conducted by Coetzee (2007) reveal not only that the PCRI items satisfy the psychometric criteria of both convergent and discriminant validity, but also that their content is commensurate with the theoretical constructs being measured.

The reliability of the PCRI was determined by means of Cronbach-Alpha coefficient. According to Anastasi (1976), a desirable reliability coefficient would fall in the range of 0.80 to 0.90. Nunnaly and Bernstein (1994) use 0.70 as a directive, whilst Bartholomew, Antonia, \& Marcia (2000) argue that between 0.80 and 0.60 is acceptable. The reliability of the 15 constructs measured by the Cronbach-Alpha was also confirmed by means of the Kaiser-Meyer-Olkin (KMO) measure of sampling adequacy and the Bartlett test of sphericity (Coetzee, 2007). As shown in Table 1, the overall average of the Cronbach-Alpha for each subscale ranges from 0.71 to 0.88 which clearly falls within the range of directives. The KMO measure for adequacy ranged from 0.79 to 0.92 for each of the subscales indicating that the sample was adequate. The Bartlett test of sphericity yielded a statistical approximate chi-square $(p<0.001)$ for each of the subscales, which also indicated the probability that the correlation matrix had significant correlation amongst the variables (Coetzee, 2007).

Since the purpose of this study was not to make individual predictions based on the PCRI, but rather to investigate broad

TABLE 2

Descriptive statistics: PCRI scales $(\mathrm{N}=2$ 997)

\begin{tabular}{lll}
\hline PCRI SCALE & MEAN & SD \\
\hline Career Preferences & & \\
\hline Stability/Expertise & 3.52 & 0.48 \\
Managerial & 2.84 & 0.77 \\
Variety/Creativity & 3.17 & 0.71 \\
Freedom/Autonomy & 2.81 & 0.70 \\
Career Values & & \\
\hline Growth/Development & 3.58 & 0.46 \\
Authority/Influence & 2.84 & 0.71 \\
Career Enablers & & \\
\hline Practical/Creative skills & 2.41 & 0.69 \\
Self/other skills & 3.40 & 0.53 \\
Career Drivers & & \\
\hline Career Purpose & 3.62 & 0.41 \\
Career Directedness & 3.01 & 0.68 \\
Career Venturing & 2.92 & 0.85 \\
Career Harmonisers & & \\
\hline Self-esteem & 3.30 & 0.51 \\
Behavioural adaptability & 3.22 & 0.54 \\
Emotional literacy & 3.05 & 0.60 \\
Social connectivity & 3.33 & 0.55 \\
\hline
\end{tabular}


TABLE 3

Mann-Whitney U-test for gender $(\mathrm{N}=2$ 997)

\begin{tabular}{|c|c|c|c|c|}
\hline PCRI SCALES & $\begin{array}{l}\text { MANN- } \\
\text { WHITNEY U }\end{array}$ & WILCOXON W & $\mathbf{Z}$ & SIG \\
\hline \multicolumn{5}{|l|}{ Career Preferences } \\
\hline Stability/Expertise & 989513.500 & 2519888.500 & -4.347 & $0.000^{* * *}$ \\
\hline Managerial & 897756.000 & 2426382.000 & -8.241 & $0.000^{\text {*** }}$ \\
\hline Variety/Creativity & 1013623.500 & 2543998.500 & -3.307 & $0.001^{*}$ \\
\hline Freedom/Autonomy & 990474.500 & 2519100.500 & -4.178 & $0.000^{* * *}$ \\
\hline \multicolumn{5}{|l|}{ Career Values } \\
\hline Growth/Development & 1022219.500 & 2550845.500 & -2.806 & $0.005^{\star \star}$ \\
\hline Authority/Influence & 1012902.000 & 2541528.000 & -3.187 & $0.001^{* *}$ \\
\hline \multicolumn{5}{|l|}{ Career Enablers } \\
\hline Practical/Creative skills & 987302.000 & 2515928.000 & -4.280 & $0.000^{\star \star \star}$ \\
\hline Self/other skills & 1059896.500 & 1836777.500 & -1.292 & 0.196 \\
\hline \multicolumn{5}{|l|}{ Career Drivers } \\
\hline Career Purpose & 1086699.500 & 1863580.500 & -.102 & .919 \\
\hline Career Directedness & 1076879.500 & 1853760.500 & -.526 & .599 \\
\hline Career Venturing & 1056552.000 & 2581683.000 & -1.363 & .173 \\
\hline \multicolumn{5}{|l|}{ Career Harmonisers } \\
\hline Self-esteem & 1007766.000 & 2538141.000 & -3.523 & $0.000^{* * \star}$ \\
\hline Behavioural adaptability & 1088329.500 & 2618704.500 & -.056 & 0.955 \\
\hline Emotional literacy & 929473.000 & 1706354.000 & -6.899 & $0.000^{* * *}$ \\
\hline Social connectivity & $9906-28.500$ & 1767509.500 & -4.325 & $0.000^{\text {*** }}$ \\
\hline
\end{tabular}

${ }^{* * *} p<0.0001{ }^{* *} p<0.01{ }^{*} p<0.05$

trends and certain relations between variables, the instrument was considered to be psychometrically acceptable.

\section{Research procedure}

Questionnaires were mailed to 60000 randomly selected students who were registered at the higher education institution for the particular year. The postal facilities of the institution were used to mail these questionnaires. Each questionnaire included a covering letter inviting subjects to participate in the study and assuring them that their individual responses would remain confidential. Participants were requested to complete the questionnaires and return them by mail to the researchers using the enclosed return envelope. A sample of 2997 usable questionnaires was returned. The questionnaires were scored electronically according to the author's instructions.

\section{Statistical analysis}

The statistical procedures chosen for this research were based on their applicability to the exploratory nature of the research design. The statistical analysis was carried out with the help of the SAS System, Version 9.1 statistical package (SAS Institute, 2003). Descriptive and inferential statistics were used to analyse the data. Since the tests for normality revealed that none of the 15 psychological career resources variables could be assumed to be normally distributed, non-parametric tests were performed in order to test for significant differences between the biographical variables and the 15 psychological career resources constructs. To determine which groups differed significantly in terms of the means of the PCRI subscales, independent-samples t-tests were applied for the gender biographical variable. Scheffés multi-comparison of means post hoc test was applied for marital status, educational level, age and race/language biographical variables. A cut-off point of $\mathrm{p} \leq 0.05$ was set for determining the significance of the findings.

\section{RESEARCH RESULTS}

\section{Descriptive statistics}

The means and standard deviations of each PCRI subscale are presented in Table 2, which shows that stability/expertise $($ mean $=3.52 ; \mathrm{SD}=0.48)$ and variety/creativity (mean $=3.17$; $\mathrm{SD}=0.71)$ are indicated as the dominant career preferences
TABLE 4

Kruskal-Wallis tests: Biographical variables (N $=2$ 997)

\begin{tabular}{|c|c|c|c|c|}
\hline PCRI SCALES & $\begin{array}{l}\text { BIOGRAPHICAL } \\
\text { VARIABLE }\end{array}$ & $\begin{array}{l}\text { CHI- } \\
\text { SQUARE }\end{array}$ & DF & SIG \\
\hline \multicolumn{5}{|l|}{ Career Preferences } \\
\hline Stability/Expertise & $\begin{array}{l}\text { Marital Status } \\
\text { Educational Level } \\
\text { Age } \\
\text { Race/Language }\end{array}$ & $\begin{array}{l}3.723 \\
14.936 \\
21.311 \\
250.298\end{array}$ & $\begin{array}{l}3 \\
6 \\
4 \\
8\end{array}$ & $\begin{array}{l}0.293 \\
0.021^{*} \\
0.000^{* * *} \\
0.000^{* * *}\end{array}$ \\
\hline Managerial & $\begin{array}{l}\text { Marital Status } \\
\text { Educational Level } \\
\text { Age } \\
\text { Race/Language }\end{array}$ & $\begin{array}{l}5.990 \\
19.742 \\
8.097 \\
167.510\end{array}$ & $\begin{array}{l}3 \\
6 \\
4 \\
8\end{array}$ & $\begin{array}{l}0.112 \\
0.003^{* *} \\
0.088 \\
0.000^{\star * *}\end{array}$ \\
\hline Variety/Creativity & $\begin{array}{l}\text { Marital Status } \\
\text { Educational Level } \\
\text { Age } \\
\text { Race/Language }\end{array}$ & $\begin{array}{l}2.791 \\
4.960 \\
10.600 \\
52.707\end{array}$ & $\begin{array}{l}3 \\
6 \\
4 \\
8\end{array}$ & $\begin{array}{l}0.425 \\
0.549 \\
0.031^{*} \\
0.000^{\star * *}\end{array}$ \\
\hline Freedom/Autonomy & $\begin{array}{l}\text { Marital Status } \\
\text { Educational Level } \\
\text { Age } \\
\text { Race/Language }\end{array}$ & $\begin{array}{l}9.357 \\
4.311 \\
4.293 \\
39.669\end{array}$ & $\begin{array}{l}3 \\
6 \\
4 \\
8\end{array}$ & $\begin{array}{l}0.025^{\star} \\
0.635 \\
0.368 \\
0.000^{\star \star \star}\end{array}$ \\
\hline \multicolumn{5}{|l|}{ Career Values } \\
\hline Growth/Development & $\begin{array}{l}\text { Marital Status } \\
\text { Educational Level } \\
\text { Age } \\
\text { Race/Language }\end{array}$ & $\begin{array}{l}5.886 \\
15.159 \\
25.699 \\
259.099\end{array}$ & $\begin{array}{l}3 \\
6 \\
4 \\
8\end{array}$ & 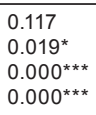 \\
\hline Authority/Influence & $\begin{array}{l}\text { Marital Status } \\
\text { Educational Level } \\
\text { Age } \\
\text { Race/Language }\end{array}$ & $\begin{array}{l}6.276 \\
8.848 \\
19.957 \\
30.884\end{array}$ & $\begin{array}{l}3 \\
6 \\
4 \\
8\end{array}$ & $\begin{array}{l}0.099 \\
0.182 \\
0.001^{\star *} \\
0.000^{\star * *}\end{array}$ \\
\hline \multicolumn{5}{|l|}{ Career Enablers } \\
\hline $\begin{array}{l}\text { Practical/Creative } \\
\text { skills }\end{array}$ & $\begin{array}{l}\text { Marital Status } \\
\text { Educational Level } \\
\text { Age } \\
\text { Race/Language }\end{array}$ & $\begin{array}{l}4.988 \\
13.257 \\
14.145 \\
62.154\end{array}$ & $\begin{array}{l}3 \\
6 \\
4 \\
8\end{array}$ & $\begin{array}{l}0.173 \\
0.039^{*} \\
0.007^{\star *} \\
0.000^{\star * *}\end{array}$ \\
\hline Self/other skills & $\begin{array}{l}\text { Marital Status } \\
\text { Educational Level } \\
\text { Age } \\
\text { Race/Language }\end{array}$ & $\begin{array}{l}4.531 \\
10.610 \\
26.985 \\
162.432\end{array}$ & $\begin{array}{l}3 \\
6 \\
4 \\
8\end{array}$ & $\begin{array}{l}0.210 \\
0.101 \\
0.000^{\star * \star} \\
0.000^{\star * \star}\end{array}$ \\
\hline \multicolumn{5}{|l|}{ Career Drivers } \\
\hline Career Purpose & $\begin{array}{l}\text { Marital Status } \\
\text { Educational Level } \\
\text { Age } \\
\text { Race/Language }\end{array}$ & $\begin{array}{l}3.490 \\
19.284 \\
8.869 \\
232.668\end{array}$ & $\begin{array}{l}3 \\
6 \\
4 \\
8\end{array}$ & $\begin{array}{l}0.322 \\
0.004^{* *} \\
0.064 \\
0.000^{\star * *}\end{array}$ \\
\hline Career Directedness & $\begin{array}{l}\text { Marital Status } \\
\text { Educational Level } \\
\text { Age } \\
\text { Race/Language }\end{array}$ & $\begin{array}{l}4.579 \\
7.140 \\
6.008 \\
83.559\end{array}$ & $\begin{array}{l}3 \\
6 \\
4 \\
8\end{array}$ & $\begin{array}{l}0.205 \\
0.308 \\
0.199 \\
0.000^{\star \star \star}\end{array}$ \\
\hline Career Venturing & $\begin{array}{l}\text { Marital Status } \\
\text { Educational Level } \\
\text { Age } \\
\text { Race/Language }\end{array}$ & $\begin{array}{l}12.247 \\
5.683 \\
1.076 \\
163.121\end{array}$ & $\begin{array}{l}3 \\
6 \\
4 \\
8\end{array}$ & $\begin{array}{l}0.007^{* *} \\
0.460 \\
0.898 \\
0.000^{* * *}\end{array}$ \\
\hline \multicolumn{5}{|l|}{ Career Harmonisers } \\
\hline Self-esteem & $\begin{array}{l}\text { Marital Status } \\
\text { Educational Level } \\
\text { Age } \\
\text { Race/Language }\end{array}$ & $\begin{array}{l}3.292 \\
14.435 \\
6.876 \\
318.386\end{array}$ & $\begin{array}{l}3 \\
6 \\
4 \\
8\end{array}$ & $\begin{array}{l}0.349 \\
0.025^{\star} \\
0.143 \\
0.000^{\star * *}\end{array}$ \\
\hline $\begin{array}{l}\text { Behavioural } \\
\text { adaptability }\end{array}$ & $\begin{array}{l}\text { Marital Status } \\
\text { Educational Level } \\
\text { Age } \\
\text { Race/Language }\end{array}$ & $\begin{array}{l}13.210 \\
3.082 \\
7.340 \\
30.396\end{array}$ & $\begin{array}{l}3 \\
6 \\
4 \\
8\end{array}$ & $\begin{array}{l}0.004^{* *} \\
0.799 \\
0.119 \\
0.000^{\star * *}\end{array}$ \\
\hline Emotional literacy & $\begin{array}{l}\text { Marital Status } \\
\text { Educational Level } \\
\text { Age } \\
\text { Race/Language }\end{array}$ & $\begin{array}{l}7.057 \\
6.333 \\
6.520 \\
11.905\end{array}$ & $\begin{array}{l}3 \\
6 \\
4 \\
8\end{array}$ & $\begin{array}{l}0.070 \\
0.387 \\
0.164 \\
0.156\end{array}$ \\
\hline Social connectivity & $\begin{array}{l}\text { Marital Status } \\
\text { Educational Level } \\
\text { Age } \\
\text { Race/Language }\end{array}$ & $\begin{array}{l}2.728 \\
6.135 \\
2.932 \\
55.475\end{array}$ & $\begin{array}{l}3 \\
6 \\
4 \\
8\end{array}$ & $\begin{array}{l}0.435 \\
0.408 \\
0.569 \\
0.000^{* * *}\end{array}$ \\
\hline
\end{tabular}

${ }^{* *} p<0.0001{ }^{* *} p<0.01{ }^{*} p<0.05$

for the total sample. In line with this observation, the sample indicated growth/development $($ mean $=3.58 ; \mathrm{SD}=0.46$ ) as their dominant career value. With respect to the career enabler subscale, the self/other skills (mean $=3.40 ; \mathrm{SD}=0.53$ ) variable obtained a higher mean score than the practical/creative skills $($ mean $=2.41 ; \mathrm{SD}=0.69)$ variable. The career purpose $($ mean $=3.62 ; \mathrm{SD}=0.41)$ and career directedness $($ mean $=3.01$ $\mathrm{SD}=0.68)$ variables obtained higher mean scores than the career venturing $($ mean $=2.92 ; \mathrm{SD}=0.85)$ variable. The variables described by the career harmonisers subscale all achieved high 
TABLE 5

Summary: Independent samples t-test for gender

\begin{tabular}{|c|c|c|c|c|c|c|c|c|}
\hline PCRI scale & Group & $\mathrm{N}$ & Mean & SD & $\mathrm{F}$ & $\mathrm{t}$ & df & Sig \\
\hline \multicolumn{9}{|l|}{ Career Preferences } \\
\hline Stability/Expertise & $\begin{array}{l}\text { Male } \\
\text { Female }\end{array}$ & $\begin{array}{l}1243 \\
1748\end{array}$ & $\begin{array}{l}2.48 \\
2.37\end{array}$ & $\begin{array}{l}0.68 \\
0.70\end{array}$ & $1.723^{* * *}$ & 4.339 & 2989 & 0.000 \\
\hline Managerial & $\begin{array}{l}\text { Male } \\
\text { Female }\end{array}$ & $\begin{array}{l}1243 \\
1748\end{array}$ & $\begin{array}{l}2.99 \\
2.74\end{array}$ & $\begin{array}{l}0.71 \\
0.79\end{array}$ & $21.703^{\star \star *}$ & 8.696 & 2992 & 0.000 \\
\hline Variety/Creativity & $\begin{array}{l}\text { Male } \\
\text { Female }\end{array}$ & $\begin{array}{l}1246 \\
1749\end{array}$ & $\begin{array}{l}3.23 \\
3.13\end{array}$ & $\begin{array}{l}0.68 \\
0.73\end{array}$ & $7.416^{* * *}$ & 3.762 & 2993 & 0.000 \\
\hline Freedom/Autonomy & $\begin{array}{l}\text { Male } \\
\text { Female }\end{array}$ & $\begin{array}{l}1244 \\
1748\end{array}$ & $\begin{array}{l}2.88 \\
2.77\end{array}$ & $\begin{array}{l}0.68 \\
0.71\end{array}$ & $2.040^{* * *}$ & 4.180 & 2990 & 0.000 \\
\hline \multicolumn{9}{|l|}{ Career Values } \\
\hline Growth/Development & $\begin{array}{l}\text { Male } \\
\text { Female }\end{array}$ & $\begin{array}{l}1242 \\
1748\end{array}$ & $\begin{array}{l}3.60 \\
3.56\end{array}$ & $\begin{array}{l}0.46 \\
0.46\end{array}$ & $1.440^{*}$ & 2.272 & 2988 & 0.023 \\
\hline Authority/Influence & $\begin{array}{l}\text { Male } \\
\text { Female }\end{array}$ & $\begin{array}{l}1242 \\
1748\end{array}$ & $\begin{array}{l}2.89 \\
2.81\end{array}$ & $\begin{array}{l}0.69 \\
0.73\end{array}$ & $5.354^{* *}$ & 3.173 & 2989 & 0.002 \\
\hline \multicolumn{9}{|l|}{ Career Enablers } \\
\hline Practical/Creative skills & $\begin{array}{l}\text { Male } \\
\text { Female }\end{array}$ & $\begin{array}{l}1243 \\
1748\end{array}$ & $\begin{array}{l}2.48 \\
2.37\end{array}$ & $\begin{array}{l}0.68 \\
0.70\end{array}$ & $1.723^{\star * *}$ & 4.339 & 2989 & 0.000 \\
\hline Self/other skills & $\begin{array}{l}\text { Male } \\
\text { Female }\end{array}$ & $\begin{array}{l}1246 \\
1749\end{array}$ & $\begin{array}{l}3.39 \\
3.41\end{array}$ & $\begin{array}{l}0.53 \\
0.52\end{array}$ & 1.644 & -1.367 & 2993 & 0.172 \\
\hline \multicolumn{9}{|l|}{ Career Drivers } \\
\hline Career Purpose & $\begin{array}{l}\text { Male } \\
\text { Female }\end{array}$ & $\begin{array}{l}1246 \\
1748\end{array}$ & $\begin{array}{l}3.62 \\
3.62\end{array}$ & $\begin{array}{l}0.42 \\
0.41\end{array}$ & 1.351 & -0.492 & 2992 & 0.623 \\
\hline Career Directedness & $\begin{array}{l}\text { Male } \\
\text { Female }\end{array}$ & $\begin{array}{l}1246 \\
1748\end{array}$ & $\begin{array}{l}3.00 \\
3.00\end{array}$ & $\begin{array}{l}0.66 \\
0.69\end{array}$ & 1.324 & -0.247 & 2992 & 0.805 \\
\hline Career Venturing & $\begin{array}{l}\text { Male } \\
\text { Female }\end{array}$ & $\begin{array}{l}1246 \\
1746\end{array}$ & $\begin{array}{l}2.95 \\
2.90\end{array}$ & $\begin{array}{l}0.84 \\
0.86\end{array}$ & 2.966 & 1.421 & 2990 & 0.155 \\
\hline \multicolumn{9}{|l|}{ Career Harmonisers } \\
\hline Self-esteem & $\begin{array}{l}\text { Male } \\
\text { Female }\end{array}$ & $\begin{array}{l}1246 \\
1749\end{array}$ & $\begin{array}{l}3.34 \\
3.27\end{array}$ & $\begin{array}{l}0.50 \\
0.52\end{array}$ & $2.640^{\star \star *}$ & 3.643 & 2993 & 0.000 \\
\hline Behavioural adaptability & $\begin{array}{l}\text { Male } \\
\text { Female }\end{array}$ & $\begin{array}{l}1246 \\
1749\end{array}$ & $\begin{array}{l}3.22 \\
3.22\end{array}$ & $\begin{array}{l}0.53 \\
0.54\end{array}$ & 0.554 & 0.166 & 2993 & 0.868 \\
\hline Emotional literacy & $\begin{array}{l}\text { Male } \\
\text { Female }\end{array}$ & $\begin{array}{l}1246 \\
1749\end{array}$ & $\begin{array}{l}2.96 \\
3.11\end{array}$ & $\begin{array}{l}0.59 \\
0.60\end{array}$ & $2.244^{\star * *}$ & -7.077 & 2993 & 0.000 \\
\hline Social connectivity & $\begin{array}{l}\text { Male } \\
\text { Female }\end{array}$ & $\begin{array}{l}1246 \\
1749\end{array}$ & $\begin{array}{l}3.28 \\
3.37\end{array}$ & $\begin{array}{l}0.57 \\
0.53\end{array}$ & $7.431^{\text {***}}$ & -4.727 & 2993 & 0.000 \\
\hline
\end{tabular}

${ }^{* * *} \mathrm{p}<0.0001{ }^{* *} \mathrm{p}<0.01{ }^{*} \mathrm{p}<0.05$

mean scores, with social connectivity (mean $=3.33$; $\mathrm{SD}=0.55)$ and self-esteem $($ mean $=3.30 ; \mathrm{SD}=0.51$ ) variables showing the highest mean scores.

\section{Mann-Whitney U-test for gender and PCRI subscales}

Table 3 shows significant differences between all the career preferences and career values variables and the gender variable. Significant differences are also indicated with regard to the practical/creative skills variable and the career harmoniser variables self-esteem, emotional literacy and social connectivity for the gender group. The career driver variables do not indicate significant differences between males and females regarding their career purpose, career directedness and career venturing. Also, no significant differences are indicated for male and female participants on the self/other skills and behavioural adaptability variables.

\section{Kruskal-Wallis test for biographical variables and PCRI subscales}

Table 4 shows significant differences between marital status and the freedom/autonomy career preference, career venturing and behavioural adaptability variables. Significant differences are also indicated between educational levels and the managerial and stability/expertise career preferences, the growth/development career value, the practical/creative skills variable, career purpose and self-esteem variables. The age groups appear to differ significantly with respect to the stability/expertise and variety/creativity career preferences and both the career values and career enablers variables. There appears to be no significant differences between the various age groups and the career drivers and career harmoniser variables. The race/language groups appear to differ significantly on all the psychological career resources variables with the exception of the emotional literacy variable. Overall,no significant differences areindicated between the emotional literacy variable and the various marital status, educational levels, age and race/language groups.

\section{Independent-samples t-tests}

The results from the independent-samples t-test shown in Table 5 indicate that both males and females obtained high mean scores on the variety/creativity career preference and growth/development career value. They also obtained high mean scores on the self/other skills variable, the career purpose and career directedness variables, and the career harmoniser variables. The results also confirm that the male and female participants do not differ significantly regarding the career drivers variables, the behavioural adaptability and self/other skills variables. However, male and female participants do appear to differ significantly with respect to the career preferences and career values variables, with the male participants showing significantly higher mean scores than the females regarding these variables. Furthermore, the male participants also show significantly higher mean scores on the practical/creativity skills and the self-esteem variables. On the other hand, female participants showed significantly higher mean scores than the men on the emotional literacy and social connectivity variables.

\section{Scheffés multi-comparison of means post hoc tests}

The results presented in Tables 6 to 10 show that the source of the significant differences indicated by the Kruskal-Wallis test could only be detected for some of the age, marital status and race/language groups. None could be detected for the educational levels groups. Key observations from the results 
TABLE 6

Summary of Scheffé multiple comparison tests:

Significant group mean differences on career preferences scale $(N=2997)$

\begin{tabular}{|c|c|c|c|c|}
\hline (I) Age & (J) Age & $\begin{array}{l}\text { Mean } \\
\text { Difference (I-J) }\end{array}$ & $\begin{array}{l}\text { Std } \\
\text { Error }\end{array}$ & Sig \\
\hline 56 years \& older & $31-40$ years & -0.32681 & 0.10680 & 0.053 \\
\hline $\begin{array}{l}\text { Race/Language } \\
\text { (I) }\end{array}$ & $\begin{array}{l}\text { Race/Language } \\
\text { (J) }\end{array}$ & $\begin{array}{l}\text { Mean Difference } \\
(I-J)\end{array}$ & Std Error & Sig \\
\hline Indian & $\begin{array}{l}\text { African Sotho } \\
\text { African Nguni } \\
\text { White English }\end{array}$ & $\begin{array}{l}-0.2142^{* * *} \\
-0.1922^{* *} \\
0.1988^{* *}\end{array}$ & $\begin{array}{l}0.03778 \\
0.03803 \\
0.04053\end{array}$ & $\begin{array}{l}0.000^{* * *} \\
0.001^{* *} \\
0.002^{* *}\end{array}$ \\
\hline $\begin{array}{l}\text { Coloured } \\
\text { English }\end{array}$ & $\begin{array}{l}\text { African Nguni } \\
\text { African Sotho }\end{array}$ & $\begin{array}{l}-0.2382^{\star *} \\
-0.2602^{\star *}\end{array}$ & $\begin{array}{l}0.04955 \\
0.04936\end{array}$ & $\begin{array}{l}0.003^{\star \star} \\
0.001^{\star \star}\end{array}$ \\
\hline $\begin{array}{l}\text { White } \\
\text { English }\end{array}$ & $\begin{array}{l}\text { African English } \\
\text { African Nguni } \\
\text { African Sotho } \\
\text { Minority } \\
\text { Indian } \\
\text { Coloured Afrikaans } \\
\text { White Afrikaans }\end{array}$ & $\begin{array}{l}-0.3412^{* *} \\
-0.3910^{* * *} \\
-0.4130^{\star \star *} \\
-0.3827^{* * *} \\
-0.1988^{* *} \\
-0.2695^{\star} \\
-0.1940^{* \star *}\end{array}$ & $\begin{array}{l}0.07040 \\
0.03042 \\
0.03010 \\
0.04357 \\
0.04053 \\
0.06224 \\
0.03104\end{array}$ & $\begin{array}{l}0.003^{* *} \\
0.000^{* * *} \\
0.000^{* * *} \\
0.000^{* * *} \\
0.002^{\star *} \\
0.017^{*} \\
0.000^{* * *}\end{array}$ \\
\hline $\begin{array}{l}\text { White } \\
\text { Afrikaans }\end{array}$ & $\begin{array}{l}\text { African Nguni } \\
\text { African Sotho } \\
\text { Minority } \\
\text { White English }\end{array}$ & $\begin{array}{l}-0.1970^{\star * *} \\
-0.2189^{* \star *} \\
-0.1886^{* *} \\
0.1940^{\star * *}\end{array}$ & $\begin{array}{l}0.02769 \\
0.02734 \\
0.04171 \\
0.03104\end{array}$ & $\begin{array}{l}0.000^{* * *} \\
0.000^{* * *} \\
0.009^{* *} \\
0.000^{* * *}\end{array}$ \\
\hline
\end{tabular}

PCRI SCALE: CAREER PREFERENCES - MANAGERIAL

\begin{tabular}{|c|c|c|c|c|}
\hline $\begin{array}{l}\text { Race/ } \\
\text { Language (I) }\end{array}$ & $\begin{array}{l}\text { Race/Language } \\
\text { (J) }\end{array}$ & $\begin{array}{l}\text { Mean } \\
\text { Difference (I-J) }\end{array}$ & $\begin{array}{l}\text { Std } \\
\text { Error }\end{array}$ & Sig \\
\hline Indian & $\begin{array}{l}\text { African Nguni } \\
\text { African Sotho } \\
\text { White English }\end{array}$ & $\begin{array}{l}.2470\left(^{\star}\right) \\
-.2708\left(^{\star}\right) \\
.2870\left(^{\star}\right)\end{array}$ & $\begin{array}{l}.06072 \\
.06031 \\
.06471\end{array}$ & $\begin{array}{l}0.036^{\star} \\
0.010^{\star *} \\
0.012^{*}\end{array}$ \\
\hline $\begin{array}{l}\text { Coloured } \\
\text { English }\end{array}$ & $\begin{array}{l}\text { African Nguni } \\
\text { African Sotho }\end{array}$ & $\begin{array}{l}-.3800\left(^{*}\right) \\
-.4039\left(^{*}\right)\end{array}$ & $\begin{array}{l}.07910 \\
.07879\end{array}$ & $\begin{array}{l}0.003^{\star *} \\
0.001^{\star *}\end{array}$ \\
\hline White English & $\begin{array}{l}\text { African Nguni } \\
\text { African Sotho } \\
\text { Minority } \\
\text { Indian } \\
\text { Coloured Afrikaans } \\
\text { White Afrikaans }\end{array}$ & $\begin{array}{l}-.5340\left(^{*}\right) \\
-.5578\left(^{*}\right) \\
-.5141\left(^{*}\right) \\
-.2870\left(^{*}\right) \\
-.4387\left(^{*}\right) \\
-.2248\left(^{*}\right)\end{array}$ & $\begin{array}{l}.04856 \\
.04805 \\
.06956 \\
.06471 \\
.09936 \\
.04955\end{array}$ & $\begin{array}{l}0.000^{* * *} \\
0.000^{* * *} \\
0.000^{* * *} \\
0.012^{*} \\
0.013^{*} \\
0.009^{* *}\end{array}$ \\
\hline White Afrikaans & $\begin{array}{l}\text { African Nguni } \\
\text { African Sotho } \\
\text { Minority } \\
\text { White English }\end{array}$ & $\begin{array}{c}-.3091\left(^{*}\right) \\
-.3330\left(^{*}\right) \\
-.2893\left(^{*}\right) \\
.2248\left(^{*}\right)\end{array}$ & $\begin{array}{l}.04421 \\
.04365 \\
.06660 \\
.04955\end{array}$ & $\begin{array}{l}0.000^{* * *} \\
0.000^{* * *} \\
0.016^{*} \\
0.009^{* *}\end{array}$ \\
\hline
\end{tabular}

PCRI SCALE: CAREER PREFERENCES - VARIETYICREATIVITY

\begin{tabular}{lllll}
\hline $\begin{array}{l}\text { Race/ } \\
\text { Language (I) }\end{array}$ & $\begin{array}{l}\text { Race/Language } \\
\text { (J) }\end{array}$ & $\begin{array}{l}\text { Mean } \\
\text { Difference } \\
(\mathbf{I}-\mathrm{J})\end{array}$ & $\begin{array}{l}\text { Std } \\
\text { Error }\end{array}$ & Sig \\
\hline White English & $\begin{array}{l}\text { African Sotho } \\
\text { Minority }\end{array}$ & $-.2446\left(^{*}\right)$ & .04574 & $0.000^{* * *}$ \\
& $-.2936\left(^{*}\right)$ & .06621 & $0.012^{* *}$
\end{tabular}

PCRI SCALE: CAREER PREFERENCES - FREEDOM/AUTONOMY

\begin{tabular}{lllll}
\hline $\begin{array}{l}\text { Race/ } \\
\text { Language (I) }\end{array}$ & $\begin{array}{l}\text { Race/Language } \\
\text { (J) }\end{array}$ & $\begin{array}{l}\text { Mean } \\
\text { Difference } \\
(\mathbf{I}-\mathrm{J})\end{array}$ & $\begin{array}{l}\text { Std } \\
\text { Error }\end{array}$ & Sig \\
\hline White English & African Nguni & $-.1854\left(^{*}\right)$ & .04541 & $0.034^{*}$ \\
& African Sotho & $-.2409\left(^{* *}\right)$ & .04494 & $0.000^{* * *}$ \\
White Afrikaans & African Sotho & $-.1746\left(^{*}\right)$ & .04082 & $0.019^{*}$ \\
\hline
\end{tabular}

${ }^{* * *} p<0.0001{ }^{* *} \mathrm{p}<0.01{ }^{*} \mathrm{p}<0.05$

shown in Tables 6 to 10 are the following: with respect to marital status, it appears that the single participants obtained significantly higher mean scores than the married participants on the career venturing variable and significantly lower mean scores than the divorced participants on the emotional literacy variable. Participants in the late life stage (56 years and older) show significantly lower mean scores than the late to early adulthood group (31-40 years) regarding the stability/expertise career preference and self-esteem variable. Participants in the early adulthood life stage ( 25 years and younger) obtained significantly lower mean scores than the late-early adulthood (midlife transition) and midlife groups (31-40 years; 41-55 years) on the growth/development career value and self/ other skills variable. On the other hand, this group obtained significantly higher mean scores than the middle and late-early adulthood groups (26-30 years; $31-40$ years) on the authority/ influence career value.
With respect to the race/language groups, the African Sothoand Nguni-speaking participants (and in particular the Sothospeaking group) obtained higher mean scores than the other race/language groups on all the psychological career resources variables. On the other hand, the white English-speaking participants overall obtained the lowest mean scores on all the PCRI variables. The white English-speaking participants also show significantly lower mean scores when compared to the African Sotho- and Nguni-speaking participants on all the career preferences, career values, career enablers and career drivers variables. In terms of the career harmoniser variables, the white English-speaking participants show significantly lower mean scores than the African groups and white Afrikaans-speaking participants with regard to the selfesteem variable. In addition, they obtained significantly lower mean scores than the Indian participants on the behavioural adaptability variable. They also obtained significantly lower mean scores than both the African and Indian groups on the social connectivity variable.

The white Afrikaans-speaking group obtained significantly lower mean scores than the African Nguni- and Sothospeaking groups on the stability/expertise, managerial and freedom/autonomy career preference variables; the growth/ development variable; the self/other skills variable, the three career drivers variables and the social connectivity variable. The coloured English-speaking participants show significantly lower mean scores than the African Sotho- and Nguni-speaking groups on the stability/expertise and managerial career preferences, the growth/development career value, and the career enabler, career purpose and career venturing variables. The Indian participants show significantly lower mean scores than the African Sotho- and Nguni-speaking participants on the stability/expertise and managerial career preference variables. The participants from the minority group obtained significantly higher mean scores than the white participants on the stability/expertise and managerial career preference variables; the growth/development career value variable; the career drivers and career enablers variables and the self-esteem variable.

TABLE 7

Summary of Scheffé multiple comparison tests:

Significant group mean differences on career values scale $(\mathrm{N}=2$ 997)

\begin{tabular}{|c|c|c|c|c|}
\hline \multicolumn{5}{|c|}{ PCRI SCALE: CAREER VALUES - GROWTH/DEVELOPMENT } \\
\hline (I) Age & (J) Age & $\begin{array}{l}\text { Mean Difference } \\
(I-J)\end{array}$ & Std Error & Sig \\
\hline 25 years \& younger & $\begin{array}{l}31-40 \text { years } \\
41-55 \text { years }\end{array}$ & $\begin{array}{l}.08489\left(^{*}\right) \\
-.09340\left(^{*}\right)\end{array}$ & $\begin{array}{l}.02166 \\
.02648\end{array}$ & $\begin{array}{l}0.004^{* *} \\
0.015^{*}\end{array}$ \\
\hline $\begin{array}{l}\text { Race/Language } \\
\text { (I) }\end{array}$ & $\begin{array}{l}\text { Race/ } \\
\text { Language (J) }\end{array}$ & $\begin{array}{l}\text { Mean Difference } \\
(I-J)\end{array}$ & Std Error & Sig \\
\hline $\begin{array}{l}\text { Coloured } \\
\text { English }\end{array}$ & $\begin{array}{l}\text { African Nguni } \\
\text { African Sotho } \\
\text { Minority }\end{array}$ & $\begin{array}{l}-.2879\left(^{*}\right) \\
-.2726\left(^{*}\right) \\
-.2678\left({ }^{*}\right)\end{array}$ & $\begin{array}{l}.04780 \\
.04761 \\
.05648\end{array}$ & $\begin{array}{l}0.000^{* * *} \\
0.000^{* * *} \\
0.004^{* *}\end{array}$ \\
\hline $\begin{array}{l}\text { White } \\
\text { English }\end{array}$ & $\begin{array}{l}\text { African English } \\
\text { African Nguni } \\
\text { African Sotho } \\
\text { Minority } \\
\text { Indian }\end{array}$ & $\begin{array}{l}-.2989\left(^{*}\right) \\
-.3375\left(^{*}\right) \\
-.3222\left(^{*}\right) \\
-.3174\left(^{*}\right) \\
-.2587\left(^{*}\right.\end{array}$ & $\begin{array}{l}.06791 \\
.02935 \\
02903 \\
.04203 \\
.03910\end{array}$ & $\begin{array}{l}0.013^{\star} \\
0.000^{\star \star *} \\
0.000^{\star * *} \\
0.000^{\star * *} \\
0.000^{\star \star *}\end{array}$ \\
\hline $\begin{array}{l}\text { White } \\
\text { Afrikaans }\end{array}$ & $\begin{array}{l}\text { African Nguni } \\
\text { African Sotho } \\
\text { Minority } \\
\text { Indian }\end{array}$ & $\begin{array}{l}-.2762\left(^{\star}\right) \\
-.2610\left(^{*}\right) \\
-.2562\left(^{*}\right) \\
-.1975\left(^{*}\right)\end{array}$ & $\begin{array}{l}.02673 \\
.02638 \\
.04024 \\
.03717\end{array}$ & $\begin{array}{l}0.000^{\star * *} \\
0.000^{\star * *} \\
0.000^{\star * *} \\
0.000^{\star * *}\end{array}$ \\
\hline \multicolumn{5}{|c|}{ PCRI SCALE: CAREER VALUES- AUTONOMY/INFLUENCE } \\
\hline (l) Age & (J) Age & $\begin{array}{l}\text { Mean Difference } \\
(\mathrm{I}-\mathrm{J})\end{array}$ & Std Error & Sig \\
\hline 25 years \& younger & $\begin{array}{l}26-30 \text { years } \\
31-40 \text { years }\end{array}$ & $\begin{array}{l}.1516\left(^{*}\right) \\
.1203\left({ }^{*}\right)\end{array}$ & $\begin{array}{l}.03922 \\
.03419\end{array}$ & $\begin{array}{l}0.005^{* *} \\
0.015^{*}\end{array}$ \\
\hline $\begin{array}{l}\text { Race/Language } \\
\text { (I) }\end{array}$ & $\begin{array}{l}\text { Race/ } \\
\text { Language } \\
(\mathrm{J})\end{array}$ & $\begin{array}{l}\text { Mean Difference } \\
(I-J)\end{array}$ & Std Error & Sig \\
\hline White English & African Nguni & $-0.19108\left(^{*}\right)$ & .04615 & $0.029^{*}$ \\
\hline
\end{tabular}

${ }^{* * *} \mathrm{p}<0.0001{ }^{* *} \mathrm{p}<0.01{ }^{*} \mathrm{p}<0.05$ 
TABLE 8

Summary of Scheffé multiple comparison tests: Significant group mean differences on career enablers scale ( $N=2997)$

\begin{tabular}{|c|c|c|c|c|}
\hline $\begin{array}{l}\text { Race/Language } \\
\text { (I) }\end{array}$ & $\begin{array}{l}\text { Race/Language } \\
\text { (J) }\end{array}$ & $\begin{array}{l}\text { Mean } \\
\text { Difference } \\
(I-J)\end{array}$ & Std Error & Sig \\
\hline African Sotho & $\begin{array}{l}\text { Coloured English } \\
\text { White English } \\
\text { White Afrikaans }\end{array}$ & $\begin{array}{l}.3602\left(^{*}\right) \\
.2274\left(^{*}\right) \\
.1657\left(^{*}\right)\end{array}$ & $\begin{array}{l}.07261 \\
.04433 \\
.04025\end{array}$ & $\begin{array}{l}0.002^{* *} \\
0.001^{* *} \\
0.031^{*}\end{array}$ \\
\hline $\begin{array}{l}\text { Coloured } \\
\text { English }\end{array}$ & $\begin{array}{l}\text { African Nguni } \\
\text { African Sotho } \\
\text { Minority }\end{array}$ & $\begin{array}{l}-.3258\left(^{*}\right) \\
-.3602\left(^{*}\right) \\
-.4336\left({ }^{*}\right)\end{array}$ & $\begin{array}{l}.07289 \\
.07261 \\
.08612\end{array}$ & $\begin{array}{l}0.011^{*} \\
0.002^{* *} \\
0.001^{* *}\end{array}$ \\
\hline $\begin{array}{l}\text { White } \\
\text { English }\end{array}$ & $\begin{array}{l}\text { African Nguni } \\
\text { African Sotho } \\
\text { Minority }\end{array}$ & $\begin{array}{l}-.1930\left(^{*}\right) \\
-.2274\left(^{*}\right) \\
-.3008\left(^{*}\right)\end{array}$ & $\begin{array}{l}.04479 \\
.04433 \\
.06411\end{array}$ & $\begin{array}{l}0.018^{\star} \\
0.001^{\star *} \\
0.005^{\star \star}\end{array}$ \\
\hline \multicolumn{5}{|c|}{ PCRI SCALE: CAREER ENABLERS - SELF/OTHER SKILLS } \\
\hline Age (I) & Age (J) & $\begin{array}{l}\text { Mean } \\
\text { Difference } \\
(I-J)\end{array}$ & Std Error & Sig \\
\hline 25 years $\&$ younger & $\begin{array}{l}31-40 \text { years } \\
41-55 \text { years }\end{array}$ & $\begin{array}{l}-0.0811^{*} \\
-0.1440^{* * *}\end{array}$ & $\begin{array}{l}0.02461 \\
0.03036\end{array}$ & $\begin{array}{l}0.029^{*} \\
0.000^{* * *}\end{array}$ \\
\hline \multirow[t]{2}{*}{$41-55$ years } & $\begin{array}{l}25 \text { years \& younger } \\
26-30 \text { years }\end{array}$ & $0.1440^{* * *}$ & 0.03036 & $0.000^{* * *}$ \\
\hline & & $0.1120^{*}$ & 0.03272 & $0.020^{*}$ \\
\hline $\begin{array}{l}\text { Race/Language } \\
\text { (I) }\end{array}$ & $\begin{array}{l}\text { Race/Language } \\
\text { (J) }\end{array}$ & $\begin{array}{l}\text { Mean } \\
\text { Difference } \\
(I-J)\end{array}$ & Std Error & Sig \\
\hline Coloured English & African Nguni & $-.2176\left(^{*}\right)$ & .05422 & $0.041^{*}$ \\
\hline White English & $\begin{array}{l}\text { African English } \\
\text { African Nguni } \\
\text { African Sotho } \\
\text { Minority } \\
\text { Indian }\end{array}$ & $\begin{array}{l}-.3178\left(^{*}\right) \\
-.3107\left(^{*}\right) \\
-.2694\left(^{*}\right) \\
-.3038\left(^{*}\right) \\
-.2512\left(^{*}\right)\end{array}$ & $\begin{array}{l}.07703 \\
.03332 \\
.03298 \\
.04769 \\
.04437\end{array}$ & $\begin{array}{l}0.030^{*} \\
0.000^{* * *} \\
0.000^{* * *} \\
0.000^{* * *}\end{array}$ \\
\hline White Afrikaans & $\begin{array}{l}\text { African Nguni } \\
\text { African Sotho } \\
\text { Minority } \\
\text { Indian }\end{array}$ & $\begin{array}{l}-.2812\left(^{*}\right) \\
-.2400\left(^{*}\right) \\
-.2744\left(^{\star}\right) \\
-.2218\left(^{\star}\right)\end{array}$ & $\begin{array}{l}.03031 \\
.02994 \\
.04564 \\
.04216\end{array}$ & $\begin{array}{l}0.000^{* * *} \\
0.000^{* * *} \\
0.000^{* * *} \\
0.001^{* *}\end{array}$ \\
\hline
\end{tabular}

${ }^{* * *} \mathrm{p}<0.0001{ }^{* *} \mathrm{p}<0.01{ }^{*} \mathrm{p}<0.05$

\section{DISCUSSION}

The main objective of this study was to explore broad trends regarding how individuals from various age, educational, marital, race and gender groups in the South African organisational context differ in terms of their psychological career resources. Overall, the findings suggest a number of significant differences between the psychological career resources of the various biographical groups. In interpreting the results, the following biographical characteristics of the sample were kept in mind: participants were predominantly from the service industry and held mostly managerial and supervisory positions. Furthermore, the sample represented predominantly full-time, employed, black single and married females in the early adulthood life stage (25-40 years) who were enrolled for undergraduate studies at the higher education institution.

\section{Gender}

The findings of this study show in line with results from other studies that females tend to rate higher than males on emotional literacy and self/other skills. Women have also been found to be more supportive, empathetic and emotionally selfaware than are men (BarOn, 1997; Brown et al., 2003). Chan and Hui (1995) found that men were more constrained in expressing their emotions and that women had a greater tendency to seek advice and social support.

\section{Marital status}

The overall psychological career resources profile of participants indicates that apart from their high sense of purpose, the participants seemingly have well-developed self/other skills, strong social connectivity, positive self-esteem and behavioural adaptability. On the other hand, their practical/creative skills, career directedness, career venturing and emotional literacy
TABLE 9

Summary of Scheffé multiple comparison tests:

Significant group mean differences on career drivers scale $(N=2997)$

\begin{tabular}{|c|c|c|c|c|}
\hline \multicolumn{5}{|c|}{ PCRI SCALE: CAREER DRIVERS- CAREER PURPOSE } \\
\hline Race/Language (I) & Race/Language (J) & $\begin{array}{l}\text { Mean } \\
\text { Difference } \\
(I-J)\end{array}$ & Std Error & Sig \\
\hline $\begin{array}{l}\text { Coloured } \\
\text { English }\end{array}$ & African Nguni & $-.1768\left(^{*}\right)$ & .04232 & $0.026^{*}$ \\
\hline $\begin{array}{l}\text { White } \\
\text { English }\end{array}$ & $\begin{array}{l}\text { African English } \\
\text { African Nguni } \\
\text { African Sotho } \\
\text { Minority } \\
\text { Indian } \\
\text { Coloured Afrikaans }\end{array}$ & $\begin{array}{l}-.3249\left(^{*}\right) \\
-.3223\left(^{*}\right) \\
-.3004\left(^{*}\right) \\
-.3352\left(^{*}\right) \\
-.2178\left(^{*}\right) \\
-.2536\left(^{*}\right)\end{array}$ & $\begin{array}{l}.05960 \\
.02581 \\
.02552 \\
.03708 \\
.03445 \\
.05247\end{array}$ & $\begin{array}{l}0.000^{* * *} \\
0.000^{* * *} \\
0.000^{* * *} \\
0.000^{* * *} \\
0.000^{* * *} \\
0.003^{* *}\end{array}$ \\
\hline $\begin{array}{l}\text { White } \\
\text { Afrikaans }\end{array}$ & $\begin{array}{l}\text { African Nguni } \\
\text { African Sotho } \\
\text { Minority }\end{array}$ & $\begin{array}{l}-.2216\left(^{*}\right) \\
-.1997\left(^{*}\right) \\
-.2345\left(^{*}\right)\end{array}$ & $\begin{array}{l}.02359 \\
.02326 \\
.03557\end{array}$ & $\begin{array}{l}0.000^{* * *} \\
0.000^{* * *} \\
0.000^{* * *}\end{array}$ \\
\hline \multicolumn{5}{|c|}{ PCRI SCALE: CAREER DRIVERS-CAREER DIRECTEDNESS } \\
\hline Race/Language (I) & Race/Language (J) & $\begin{array}{l}\text { Mean } \\
\text { Difference } \\
\text { (I-J) }\end{array}$ & Std Error & Sig \\
\hline White English & $\begin{array}{l}\text { African Nguni } \\
\text { African Sotho } \\
\text { Minority } \\
\text { Indian }\end{array}$ & $\begin{array}{l}-.2974\left(^{*}\right) \\
-.3235\left(^{*}\right) \\
-.3752\left(^{*}\right) \\
-.2468\left(^{*}\right)\end{array}$ & $\begin{array}{l}.04350 \\
.04300 \\
.06250 \\
.05806\end{array}$ & $\begin{array}{l}0.000^{* * *} \\
0.000^{* * *} \\
0.000^{* * *} \\
0.021^{*}\end{array}$ \\
\hline White Afrikaans & $\begin{array}{l}\text { African Nguni } \\
\text { African Sotho } \\
\text { Minority }\end{array}$ & $\begin{array}{l}-.1733\left(^{*}\right) \\
-.1994\left(^{*}\right) \\
-.2511\left(^{*}\right)\end{array}$ & $\begin{array}{l}.03975 \\
.03920 \\
.05995\end{array}$ & $\begin{array}{l}0.015^{\star} \\
0.001^{\star *} \\
0.025^{\star}\end{array}$ \\
\hline \multicolumn{5}{|c|}{ PCRI SCALE: CAREER DRIVERS-CAREER VENTURING } \\
\hline Race/Language (I) & Race/Language (J) & $\begin{array}{l}\text { Mean } \\
\text { Difference } \\
(I-J)\end{array}$ & Std Error & Sig \\
\hline Coloured English & $\begin{array}{l}\text { African Nguni } \\
\text { African Sotho }\end{array}$ & $\begin{array}{l}-3730\left(^{*}\right) \\
-.3716\left({ }^{*}\right)\end{array}$ & $\begin{array}{l}.08874 \\
.08836\end{array}$ & $\begin{array}{l}0.024^{*} \\
0.024^{*}\end{array}$ \\
\hline White English & $\begin{array}{l}\text { African Nguni } \\
\text { African Sotho } \\
\text { Minority }\end{array}$ & $\begin{array}{l}-.4509\left(^{*}\right) \\
-.4494\left(^{*}\right. \\
-.4498\left(^{*}\right)\end{array}$ & $\begin{array}{l}.05413 \\
.05350 \\
.07776\end{array}$ & $\begin{array}{l}0.000^{* * *} \\
0.000^{* * *} \\
0.000^{* * *}\end{array}$ \\
\hline White Afrikaans & $\begin{array}{l}\text { African Nguni } \\
\text { African Sotho } \\
\text { Minority }\end{array}$ & $\begin{array}{l}-.4362\left(^{*}\right) \\
-.4348\left({ }^{*}\right) \\
-.4352\left({ }^{*}\right)\end{array}$ & $\begin{array}{l}.04946 \\
.04878 \\
.07458\end{array}$ & $\begin{array}{l}0.000^{* * *} \\
0.000^{* * *} \\
0.000^{* * *}\end{array}$ \\
\hline $\begin{array}{l}\text { Marital Status } \\
\text { (I) }\end{array}$ & $\begin{array}{l}\text { Marital Status } \\
(\mathrm{J})\end{array}$ & $\begin{array}{l}\text { Mean } \\
\text { Difference } \\
(I-J)\end{array}$ & Std Error & Sig \\
\hline Single & Married & $.10095\left(^{*}\right)$ & .03227 & $0.021^{*}$ \\
\hline
\end{tabular}

${ }^{* * *} p<0.0001{ }^{* *} p<0.01{ }^{*} p<0.05$

appear to be slightly out of balance. These findings suggest an apparent lack of skills in exploring, setting and implementing future career options in creative and innovative ways. In terms of career venturing, single participants seem to be more oriented towards taking risks in finding and experimenting with new career opportunities than married participants. In this regard, Coetzee and Schreuder (2008) report findings which indicate that single people are more willing to explore career opportunities which allow them to develop their skills and talents in challenging environments. On the other hand, married people appear to have a stronger need for careers that offer them steady and stable employment and benefit packages (Coetzee \& Schreuder, 2008; Kniveton, 2004). In addition, the single participants in the study displayed lower ratings for emotional literacy than the divorced participants. This finding is similar to the findings of research conducted by Coetzee, Jansen, and Muller (2008) which show that people who are divorced or separated appear to have overall higher emotional coping resources than single, married and widowed individuals.

\section{Educational levels}

The findings indicate significant differences between participants' educational levels and the managerial and stability/expertise career preferences, the growth/development career value, their practical/creative skills, career purpose and self-esteem. However, the source of these differences could not be detected by the empirical analyses. Coetzee and Schreuder 
TABLE 10

Summary of Scheffé multiple comparison tests:

Significant group mean differences on career harmonisers scale $(\mathrm{N}=2997)$

\begin{tabular}{|c|c|c|c|c|}
\hline \multicolumn{5}{|c|}{$\begin{array}{l}\text { PCRI SCALE: CAREER HARMONISERS-SELF-ESTEEM } \\
\end{array}$} \\
\hline (I) Age & (J) Age & $\begin{array}{l}\text { Mean } \\
\text { Difference (I-J) }\end{array}$ & Std Error & Sig \\
\hline \multicolumn{2}{|c|}{56 years \& older $31-40$ years } & -0.32681 & 0.10680 & $0.053^{*}$ \\
\hline $\begin{array}{l}\text { Race/ } \\
\text { Language (I) }\end{array}$ & Race/Language (J) & $\begin{array}{l}\text { Mean } \\
\text { Difference (I-J) }\end{array}$ & Std Error & Sig \\
\hline African Nguni & $\begin{array}{l}\text { Indian } \\
\text { Coloured English } \\
\text { Coloured Afrikaans } \\
\text { White English } \\
\text { White Afrikaans }\end{array}$ & $\begin{array}{l}.1895\left(^{\star}\right) \\
.2717\left(^{*}\right) \\
.2776\left(^{*}\right) \\
.4691\left(^{*}\right) \\
.3066\left(^{*}\right)\end{array}$ & $\begin{array}{l}.03962 \\
.05172 \\
.06330 \\
.03174 \\
.02890\end{array}$ & $\begin{array}{l}0.004^{* *} \\
0.001^{* *} \\
0.014^{* *} \\
0.000^{* * *} \\
0.000^{* \star *}\end{array}$ \\
\hline African Sotho & $\begin{array}{l}\text { Indian } \\
\text { Coloured English } \\
\text { Coloured Afrikaans } \\
\text { White English } \\
\text { White Afrikaans }\end{array}$ & $\begin{array}{l}.1868\left(^{*}\right) \\
.2690\left(^{*}\right) \\
.2750\left(^{*}\right) \\
.4664\left(^{\star}\right) \\
.3039\left(^{*}\right)\end{array}$ & $\begin{array}{l}.03936 \\
.05152 \\
.06313 \\
.03141 \\
.02853\end{array}$ & $\begin{array}{l}0.004^{* *} \\
0.001^{* *} \\
0.015^{*} \\
0.000^{* \star *} \\
0.000^{* \star *}\end{array}$ \\
\hline Minority & $\begin{array}{l}\text { Coloured English } \\
\text { Coloured Afrikaans } \\
\text { White English } \\
\text { White Afrikaans }\end{array}$ & $\begin{array}{l}.2781\left(^{*}\right) \\
.2841\left(^{*}\right) \\
.4755\left(^{*}\right) \\
.3130\left(^{*}\right)\end{array}$ & $\begin{array}{l}.06112 \\
.07119 \\
.04548 \\
.04355\end{array}$ & $\begin{array}{l}0.008^{\star \star} \\
0.044^{*} \\
0.000^{\star \star \star} \\
0.000^{\star \star \star}\end{array}$ \\
\hline White English & $\begin{array}{l}\text { African English } \\
\text { African Nguni } \\
\text { African Sotho } \\
\text { Minority } \\
\text { Indian } \\
\text { White Afrikaans }\end{array}$ & $\begin{array}{l}-.4580\left(^{*}\right) \\
-.4691\left(^{*}\right) \\
-.4664\left(^{*}\right) \\
-.4755\left(^{*}\right) \\
-.2796\left(^{*}\right) \\
-.1625\left(^{*}\right)\end{array}$ & $\begin{array}{l}.07349 \\
.03174 \\
.03141 \\
.04548 \\
.04224 \\
.03240\end{array}$ & $\begin{array}{l}0.000^{\star * *} \\
0.000^{\star * *} \\
0.000^{\star * *} \\
0.000^{\star * *} \\
0.000^{\star * *} \\
0.000^{\star * *}\end{array}$ \\
\hline \multicolumn{5}{|c|}{ PCRI SCALE: CAREER HARMONISERS-BEHAVIOURAL ADAPTABILITY } \\
\hline $\begin{array}{l}\text { Race/ } \\
\text { Language (I) }\end{array}$ & Race/Language (J) & $\begin{array}{l}\text { Mean } \\
\text { Difference (I-J) }\end{array}$ & Std Error & Sig \\
\hline \multirow{3}{*}{$\begin{array}{l}\text { Indian } \\
\text { PCRI SCALE: CA } \\
\begin{array}{l}\text { Marital Status } \\
\text { (I) }\end{array}\end{array}$} & White English & $.1998\left(^{*}\right)$ & .04685 & $0.020^{*}$ \\
\hline & AREER HARMONISE & 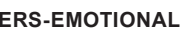 & & \\
\hline & Marital Status (J) & $\begin{array}{l}\text { Mean } \\
\text { Difference (I-J) }\end{array}$ & Std Error & Sig \\
\hline \multirow{3}{*}{$\begin{array}{l}\text { Single } \\
\text { PCRI SCALE: C } \\
\text { Race/ } \\
\text { Language (I) } \\
\end{array}$} & Divorced & $-.13695\left(^{*}\right)$ & .04772 & $0.042^{*}$ \\
\hline & ER HARIVION & L & NECCIIVI & \\
\hline & Race/Language (J) & $\begin{array}{l}\text { Mean } \\
\text { Difference (I-J) }\end{array}$ & Std Error & Sig \\
\hline White English & $\begin{array}{l}\text { African Nguni } \\
\text { African Sotho } \\
\text { Indian }\end{array}$ & $\begin{array}{l}.1810\left(^{*}\right) \\
-.1711\left(^{*}\right) \\
-.2158\left({ }^{*}\right)\end{array}$ & $\begin{array}{l}.03540 \\
.03503 \\
.04712\end{array}$ & $\begin{array}{l}0.001^{\star *} \\
0.003^{\star \star} \\
0.007^{\star \star}\end{array}$ \\
\hline White Afrikaans & African Nguni & $-.1298\left(^{\star}\right)$ & .03223 & $0.040^{*}$ \\
\hline
\end{tabular}

${ }^{* * *} p<0.0001{ }^{* *} p<0.01{ }^{*} p<0.05$

(2008) report in this regard that people with an undergraduate qualification seem to be more job oriented and are attracted to careers that afford them the opportunity to apply and develop their own skills in positions of power and influence. On the other hand, people with a post-graduate qualification seem to have a higher sense of career calling and are more interested in applying their expertise where they can help make a difference in their own and other's lives.

\section{Age}

The results of this study highlight a number of findings of particular interest. First, the participants as a group show a predominant preference for the stability/expertise and variety/creativity career preferences as well as for the growth/ development career value. This finding is rather interesting, considering that participants predominantly hold positions at senior, middle and supervisory levels. One would tend to expect a higher preference for the managerial and freedom/ autonomy career preferences and authority/influence career value as indicated by research conducted by Kniveton (2004) and Schein (1996). Since participants are occupying the managerial and supervisory positions at a relatively young age, their preferences may be an indication of their need to establish themselves in their positions and to develop their expertise through further development and growth opportunities. Adults in the early adulthood life stage were found to have a higher need for further growth and development than adults in the midlife and late-life stages (Levinson cited in Greenhaus, Callanan, \& Godshalk, 2000, p. 110). The middle and late-early adulthood life stages (26-30 years; $31-40$ years) are the periods during which the life structure of young adults become more stable as they begin to settle down, become committed to contributing towards an occupation, a company or a person and start to establish socially supportive networks (Greenhaus et al., 2000). Their need for developing expertise by means of further growth and learning opportunities are therefore higher during these particular life stages.

The second finding of interest is that the participants in the early adulthood life stage ( 25 years and younger) have a higher preference than participants in the late-early adulthood and midlife stages (31-40 years; 41-55 years) for the autonomy/ influence career value than for the growth/development career value. Similar findings are reported by Coetzee and Schreuder (2008), which indicate that people in the early adulthood life stage (25 years and younger) prefer work situations in which they will be as far as possible, free of organisational constraints and restrictions. According to Levinson (cited in Greenhaus et al., 2000, p. 110), young adults in the early adulthood life stage are at a stage in their lives when they emerge from adolescence and try to create a niche for themselves in adult society by being predominantly focused on keeping their options open and exploring various occupational roles. Their need for autonomy and independence is therefore often stronger at the age of 25 years and younger than their need to contribute towards a broader community.

\section{Race/Language}

In terms of the career harmonisers, the findings regarding the significantly higher positive self-esteem and social connectivity of the African participants in comparison with the significantly lower ratings of the white participants are in line with the findings of a study conducted by Motileng, Wagner, and Cassimjee (2006). Their studies indicated that employment equity and affirmative action strategies have improved black persons' self-esteem as these strategies seem to present them with the chance to express the capabilities, potentialities or talents they have. Positive self-esteem has been found to enable people to become pro-active agents in their actions and to increase their openness to new learning. Participation in new learning leads to greater self-awareness and a sense of purpose, and provides opportunities for the development of supportive interpersonal relationships (Borgen, Amundson, \& Reuter, 2004). In this regard, the findings indicated for the white participants a significantly lower sense of career purpose, career directedness and career venturing along with a significantly lower preference than the African participants for the managerial, stability/expertise and freedom/autonomy career preferences and the growth/development career value. These findings could suggest that white participants are not currently considering new or alternative job opportunities or that they may be experiencing uncertainty about their future career prospects. The lower ratings for the career preferences and values can most probably be attributed to the current employment equity context which promotes employment and career advancement opportunities for Africans and other black minority groups.

\section{CONCLUSION AND RECOMMENDATIONS}

The findings of this study have implications for the individual employee and the organisation. Employees need to become aware of how their particular life stage (age), marital status, educational level, gender and race relate to their career preferences and career values, and the strengths or weaknesses of their psychological career resources profile and subsequent experiences of psychological career success. Organisations must take cognisance of how the particular socio-cultural 
context in which individuals pursue their careers may influence the strength and orientation of their employees' psychological career resources profiles and their subsequent long-term employability. According to Hall and Chandler (2005), contextual forces such as socio-economic, demographic, economic or socio-political trends either facilitate or hinder individual career agency and the ability to enact a career calling. Helping people to tap into their internal (psychological) career resources will increasingly form a critical component of the career counselling and guidance service that organisations provide to their employees.

Since the present study has been limited to participants predominantly employed in the service industry, the findings cannot be generalised to other occupational contexts. Furthermore, given the exploratory nature of the research design, this study can yield no statements about causation. Associations between the biographical variables and participants' psychological career resources have therefore been interpreted rather than established. These findings therefore need to be replicated with broader samples across various occupational groups and economic sectors before conclusions can be drawn about the relationships between psychological career resources and individuals' gender, age, race, marital status and educational level. However, given that virtually no research has been undertaken on the psychological career resources of South Africa's workforce, the results of this study did provide new knowledge and valuable data on empirically significant differences and commonalities amongst the psychological career resources of a multi-cultural group of respondents employed in the South African organisational context.

\section{REFERENCES}

Allen, T.J. \& Katz, R. (1992). Age, education and the technical ladder. IEEE Transactions on Engineering Management, 39(3), 237-245.

Anastasi, A. (1976). Psychological testing. New York: McMillan.

Arthur, M.B., Khapova, S.N. \& Wilderom, C.P.M. (2005). Career success in a boundaryless career world. Journal of Organisational Behaviour, 26(1), 177-202.

BarOn, R. (1997). EQi: BarOn Emotional Quotient Inventory User's Manual. Toronto: Multi-Health Systems, Inc.

Bartholomew, K., Antonia, J.Z. \& Marcia, J.E. (2000). Coded semi-structured interviews in psychological research. In H.T. Resi \& C.M. Judd (Eds.), Handbook of research methods in social and personality psychology. Cambridge: Cambridge University Press, pp. 286-312.

Baruch, Y. (2004). Transforming careers: From linear to multidirectional career paths. Career Development International, 9(1), 58-73.

Battle, J. (1992). Culture-free self-esteem inventories for children and adults. Austin: Pro-Ed.

Bay, S.G.K. \& Lim, K.M. (2006). Correlations of multiple intelligences and emotional intelligence: A closer analysis of theoretical assumptions. The Korean Journal of Thinking $\mathcal{E}$ Problem Solving, 16(1), 53-64.

Bellah, R.N., Sullivan, W.M., Tipton, S.M., Madsen, R. \& Swindler, A. (1996). Habits of the heart: Individualism and commitment in American life. Berkeley: University of California Press.

Borgen, W.A., Amundson, N.E. \& Reuter, J. (2004). Using portfolios to enhance career resilience. Journal of Employment Counseling, 41(2), 50-59.

Briscoe, J.P. \& Hall, D.T. (1999, Autumn). Grooming and picking leaders using competency frameworks: Do they work? An alternative approach and new guidelines for practice. Organisational Dynamics, 28(2), 37-52.

Brousseau, K.R. (1990). Career dynamics in the baby boom and baby bust era. Journal of Organisational Change Management, 3(3), 46-58.
Brown, C., George-Curran, R. \& Smith, M.L. (2003). The role of emotional intelligence in the career commitment and decision-making process. Journal of Career Assessment, 11(4), 379-392.

Chan, D.W. \& Hui, E.K.P. (1995). Burnout and coping among Chinese secondary school teachers in Hong Kong. British Journal of Educational Psychology, 65(1), 15-25.

Coertse, S. \& Scheepers, J.M. (2004). Some personality and cognitive correlates of career maturity. SA Journal of Industrial Psychology, 30(2), 56-73.

Coetzee, M. (2006). Career planning in the 21st century. Cape Town: Juta.

Coetzee, M. (2007). Exploratory factor analyses of the Psychological Career Resources Inventory. Unpublished Research Report, Department of Industrial and Organisational Psychology, University of South Africa, Pretoria.

Coetzee, M., Jansen, C.A. \& Muller, H. (2008). Stress, coping resources and personality types: An exploratory study of South African teachers. Unpublished Research Report, Department of Industrial and Organisational Psychology, University of South Africa, Pretoria.

Coetzee, M. \& Roythorne-Jacobs, H. (2007). Career counseling and guidance in the workplace: A manual for career practitioners. Cape Town: Juta.

Coetzee, M. \& Schreuder, A.M.G. (2008). A multi-cultural investigation of students' career anchors at a South African higher education institution. SA Journal of Labour Relations, 32(2), (in press).

Crites, J.O. (1974). Career counseling: A review of major approaches. Counseling Psychologist, 4(3), 3-23.

Derr, C.B. (1986). Managing the new careerists. San Francisco: Jossey-Bass.

Dooley, D. (1995). Social research methods. Upper Saddle River: Prentice Hall.

Driver, M.J. (1982). Career concepts: A new approach to research. In R. Katz (Ed.), Career issues in human resource management (pp. 23-32). Englewood Cliffs: Prentice Hall.

Driver, M.J. \& Brousseau, K.R. (1988). Four career concepts. Santa Monica: Decision Dynamics Corporation.

Ebberwein, C.A., Krieshok, T.S., Ulven, J.C. \& Prosser, E.C. (2004). Voices in transition: Lessons on career adaptability. The Career Development Quarterly, 52(4), 292-308.

Emmerling, R.J. \& Cherniss, C. (2003). Emotional intelligence and the career choice process. Journal of Career Assessment, 11(2), 153-167.

Ensel, W.M. \& Lin, N. (1991). The life stress paradigm and psychological distress. Journal of Health and Social Behaviour, 32(4), 321-341.

Erdoğmus, N. (2003). Career orientations of salaried professionals: The case of Turkey. Career Development International, 9(2), 153-175.

Gardner, H. (1983). Frames of Mind. New York: Basic Books.

Garrety, K., Badham, R., Morrigan, V., Rifkin, S. \& Zanko, M. (2003). The use of personality typing in organizational change: Discourse, emotions and the reflective subject. Human Relations, 56(2), 211-235.

Göckel, A. (2004). The trend toward spirituality in the workplace: Overview and implications for career counseling. Journal of Employment Counseling, 41(2), 156-167.

Greenhaus, J.H., Callanan, G.A. \& Godshalk, V.M. (2000). Career Management. New York: Dryden.

Gowan,M.A.,Craft, S.L.S.\&Zimmermann, R.A. (2000). Response to Work Transitions by United States Army Personnel: The Effects of Self-Esteem, Self-Efficacy, and Career Resilience. Psychological Reports, 86 (3 Pt 1), 911-921.

Gunz, H.P. \& Heslin, P.A. (2005). Reconceptualising career success. Journal of Organisational Behaviour, 26(2), 105-111.

Hall, D.T. (2002). Careers in and out of organizations. Thousand Oaks: Sage.

Hall, D.T. \& Briscoe, J. (2004). Becoming protean: Individual and experiential factors in adapting to the new career. Working paper, 
Boston University Executive Development Roundtable Technical Report.

Hall, D.T. \& Chandler, D.E. (2005). Psychological success: When the career is a calling. Journal of Organisational Behaviour, 26(2), 155-176

Hammer, A.L. (1988). Manual for the Coping Resources Inventory. Palo Alto: Consulting Psychologists Press.

Hansen, L.S. (1997). Integrative life planning: Critical tasks for career development and changing life patterns. San Francisco: Jossey-Bass.

Herr, E.L., Cramer, S.H. \& Niles, S.G. (2004). Career guidance and counseling through the lifespan. London: Prentice Hall.

Hewitt, J.P. (2002). The social construction of self-esteem. In C.R Snyder \& S.J. Lopez (Eds.), Handbook of positive psychology (pp. 135-147). New York: Oxford University Press.

Higgins, M. (2001). Changing careers: The effects of social context. Journal of Organisational Behaviour, 22(1), 595-618.

Hollenbeck, G.P. \& Hall, D.T. (2004). Self-confidence and leader performance. Organisational Dynamics, 33(3), 254-269.

Igbaria, M., Greenhaus, J.H. \& Parasuraman, S. (1991). Career orientations of MIS employees: An empirical analysis. MIS Quarterly, 15(2), 151-169.

Kidd, J.M. (2004). Emotion in career contexts: Challenges for theory and research. Journal of Vocational Behaviour, 64(1), $441-454$.

Kim, N. (2005). Organisational interventions influencing employee career development preferred by different career success orientations. International Journal of Training and Development, 9(1), 47-61.

Kniveton, B.H. (2004). Managerial career anchors in a changing business environment. Journal of European Industrial Training, 28 (7), 564-573.

Lounsbury, J.W., Loveland, J.M., Sundstrom, E.D., Gibson, L.W., Drost, A.W. \& Hamrick, F.L. (2003). Journal of Career Assessment, 11(3), 287-307.

Marshall, V. \& Bonner, D. (2003). Career anchors and the effects of downsizing: Implications for generations and cultures at work: A preliminary investigation. Journal of European Industrial Training, 27(6), 281-291.

Motileng, B.B., Wagner, C. \& Cassimjee, N. (2006). Black middle managers' experience of Affirmative Action in a media company. SA Journal of Industrial Psychology, 32(1), 11-16.

Nunnaly, J.C. \& Bernstein, I.H. (1994). Psychometric theory. New York: McGraw-Hill.
Phillips, S.D., Christopher-Sisk, E.K. \& Gravino, K.L. (2001). Making career decisions in a relational context. The Counseling Psychologist, 29(2), 193-213.

Salkind, N.J. (1997). Exploring research. Fort Worth: Harcourt Brace.

SAS. (2003). Statistical Analysis System software package. Cary: SAS Institute.

Savickas, M.L. (2000). Renovating the psychology of careers for the twenty-first century. In A. Collin \& R.A. Young (Eds.), The future of career. Cambridge: Cambridge University.

Schein, E.H. (1996). Career anchors revisited. Implications for career development in the 21st century. Academy of Management Executive, 10(4), 80-88.

Schneer, J.A. \& Reitman, F. (1994). The importance of gender in mid-career: A longitudinal study of MBA's. Journal of Organisational Behaviour, 15(1), 199-207.

Schreuder, A.M.G. \& Coetzee, M. (2006). Careers: An organizational perspective. Cape Town: Juta.

Shaughnessy, J.J. \& Zechmeister, E.B. (2003). Research methods in psychology. New York: McGraw-Hill.

Sternberg, R.J. (1985). Beyond IQ: A triachic theory of human intelligence. Cambridge: Cambridge University Press.

Sternberg, R.J. (1999). Successful intelligence: Finding a balance. Trends in Cognitive Sciences, 3(1), 436-442.

Van Dam, K. (2004). Antecedents and consequences of employability orientation. European Journal of Work and Organisational Psychology, 13(1), 29-51.

Van der Heijde, C.M. \& Van der Heijden, B.I.J.M. (2006). A competence-based and multidimensional operationalisation of employability. Human Resource Management, 45(3) 449-476.

Weiss, J.W., Skelley, M.F., Freund, A.M., Haughey, J.C. \& Hall D.T. (2004). Calling, new careers and spirituality: A reflective perspective for organizational leaders and professionals. Research in Ethical Issues in Organisations, 5(1), 175-201.

Wiese, B.S., Freund, A.M. \& Baltes, P.B. (2002). Subjective career success and emotional wellbeing: longitudinal predictive power of selection, optimization and compensation. Journal of Vocational Behaviour, 60(1), 321-335.

Wrzesniewski, A., McCauley, C.R., Rozin, P. \& Schwartz, B (1997). Jobs, careers and callings: People's relations to their work. Journal of Research in Personality, 31(1), 21-33. 